\title{
Wavefront properties of the anterior and posterior corneal surface after transepithelial photorefractive keratectomy in myopia
}

\author{
LEI XI ${ }^{1,2}$ \\ ${ }^{1}$ Department of Ophthalmology, Peking University International Hospital, Beijing 102206, P.R. China; \\ ${ }^{2}$ Division of Experimental Vitreoretinal Surgery, Institute for Ophthalmic Research, Centre for Ophthalmology, \\ University of Tuebingen, D-72076 Tuebingen, Germany
}

Received April 9, 2019; Accepted October 16, 2019

DOI: $10.3892 /$ etm.2019.8338

\begin{abstract}
The present study aimed to evaluate the changes of corneal higher-order aberrations (HOAs) after transepithelial photorefractive keratectomy (TransPRK) in patients with myopia. A total of 80 eyes of 80 patients with myopia with or without astigmatism were evaluated. Prior to and 1 year after the surgery, uncorrected and corrected distance visual acuity in $\log$ MAR were assessed. Wavefront aberrations on the anterior surface of the cornea, as well as the posterior and total cornea, were measured via Pentacam. The HOA of the anterior surface and total values of spherical and coma aberrations increased significantly $(\mathrm{P}<0.001)$. However, HOAs of the anterior surface, and posterior and total values of the trefoil were not significantly changed $(\mathrm{P}=0.442,0.805$ and 0.936 , respectively). A significant correlation of the pre-operative mean spherical equivalent refraction (MSER), astigmatism and central corneal ablation depth (CCAD) with the changes in coma aberration $(r=0.268, P=0.016 ; r=0.260, P=0.020$; $\mathrm{r}=0.323, \mathrm{P}=0.004$, respectively) and $\mathrm{HOA}(\mathrm{r}=0.554, \mathrm{P}<0.001$; $\mathrm{r}=0.312, \mathrm{P}=0.005 ; \mathrm{r}=0.583, \mathrm{P}<0.001$, respectively) of the anterior surface of the cornea was determined. Furthermore, a significant correlation of the pre-operative MSER and CCAD with the changes in spherical aberration of the anterior corneal surface $(\mathrm{r}=0.462, \mathrm{P}<0.001 ; \mathrm{r}=0.510, \mathrm{P}<0.001$, respectively) was obtained. In conclusion, TransPRK offers an effective option for improving visual function in patients with myopia. The anterior corneal HOA, as well as spherical and coma aberrations, were significantly increased post-operatively. The HOA of the posterior cornea was not significantly changed. The change in the trefoil aberration was significantly correlated
\end{abstract}

Correspondence to: Dr Lei Xi, Division of Experimental Vitreoretinal Surgery, Institute for Ophthalmic Research, Centre for Ophthalmology, University of Tuebingen, Breuningerbau, Schleichstr, 12/1, D-72076 Tuebingen, Germany

E-mail: everydayxilei@163.com

Key words: transepithelial photorefractive keratectomy, myopia, wavefront aberrations, cornea with pre-operative astigmatism, but the pre- and post-operative values were not significantly different.

\section{Introduction}

Among the refractive surgery treatments, laser-mediated corneal epithelial removal, known as transepithelial photorefractive keratectomy (TransPRK), was performed in the 1990s (1). Since then, the epithelium has been removed using excimer laser photo therapeutic keratectomy followed by refractive ablation of the stroma (two-step approach). The two-step technique was not widely used due to the long intervention time, the uneven epithelial removal and treatment inaccuracy $(2,3)$.

More recently, with the development of the newer generation of faster lasers and improved ablation algorithms, a single-step treatment TransPRK was developed on the Schwind Amaris platform (Schwind eye-tech-solutions $\mathrm{GmbH}$ ). This one-step approach allows for simultaneous ablation of the epithelium and the stroma with little trauma to the eye. Studies using high-frequency digital ultrasonography have demonstrated that the corneal epithelium does not have a uniform thickness (4). The single-step ablation profile was generated from the literature targets of $55 \mu \mathrm{m}$ centrally and $65 \mu \mathrm{m}$ peripherally, using theoretical simulations for the scope of ablation optical zone (OZ) (2).

Several studies have determined increased higher-order wavefront aberrations (HOAs) after corneal refractive surgery, including PRK (5-7). However, to date, only a few studies have reported on HOAs associated with single-step TransPRK in myopia. Adib-Moghaddam et al (8) indicated that corneal spherical, coma and trefoil aberrations were significantly improved at 18 months after single-step TransPRK. Other studies comparing TransPRK with alcohol-assisted PRK indicated that the differences between the pre- and post-operative HOAs of the same type of surgery were not significant $(9,10)$. The present study evaluated the changes of corneal HOAs after TransPRK in myopia and the association between post-operative changes in HOAs and pre-operative mean spherical equivalent refraction (MSER), astigmatism and central corneal ablation depth (CCAD). HOAs in the eye are closely linked to visual quality after refractive surgery. The present study investigated the 
changes in post-operative corneal HOAs and the correlation between changes in HOA and the pre-operative diopter (D). It is important to predict post-operative corneal HOA changes and visual quality.

\section{Materials and methods}

Patient population and study design. The present retrospective study included 80 eyes of 80 patients with myopia who underwent single-step TransPRK between June 2015 and July 2017 at the Department of Ophthalmology of Peking University International Hospital (Beijing, China).

The inclusion criteria were as follows: Age $>18$ years, primary myopia with astigmatism no higher than $3 \mathrm{D}$, stable refraction for at least 12 months, discontinued contact lens use for at least 2-4 weeks (depending on the type of lens) prior to the surgery, corrected distance visual acuity (CDVA) of 20/25 or better. Exclusion criteria were abnormal or keratoconic topography, post-operative corneal bed thickness of $<325 \mu \mathrm{m}$, dry eye and other types of diagnosed ocular disease, a history of ocular trauma and pregnancy.

Pre-operative examination. Pre-operative examination included uncorrected distance visual acuity (UDVA), CDVA, manifest and cycloplegic refraction, intraocular pressure, slit lamp biomicroscopy, corneal topography, dilated fundus evaluation and ultrasound corneal pachymetry.

Wavefront measurement. Wavefront errors in each eye were measured pre-operatively and at 1 year after single-step TransPRK using a rotating Scheimpflug Camera (Pentacam Scheimpflug topography; Oculus). Patients with no haze and without dry eye were recruited for the study and the examined eye was selected at random. The examination was performed in a dark room $(11,12)$. HOAs with a $6.0-\mathrm{mm}$ analysis diameter were calculated separately for the anterior and posterior corneal surfaces and for the total cornea. The root-mean-square $(\mu \mathrm{m})$ of coma $\left(Z_{31}\right)$, trefoil $\left(Z_{33}\right)$ and spherical aberration $\left(Z_{40}\right)$ were analyzed, as they are clinically significant in terms of visual performance (7). The values of each HOA (coma, trefoil, spherical and total HOA) were determined at the post- and pre-operative stage.

Surgical technique. All surgeries were performed using an Amaris 500E excimer laser platform (Schwind eye-tech-solutions $\mathrm{GmbH}$ ) with its integrated ORK-CAM software. The epithelium and stroma were ablated in a single step. For each treatment, the epithelial thickness profile was $55 \mu \mathrm{m}$ centrally and $65 \mu \mathrm{m}$ peripherally based on population statistics. The ablation plan provides an even application of laser energy on the surface of the entire cornea and the OZ diameter was $6.30 \mathrm{~mm}$.

After the surgery, the cornea was irrigated with a cooled balanced salt solution and a bandage contact lens was applied.

Post-operative medication and protocol. After the surgery, the patients were instructed to use $0.5 \%$ levofloxacin (Cravit; Santen, Inc.) four times daily for one week, $0.1 \%$ fluorometholone drops (Allergan, Inc.) four times daily (tapered over 12 weeks) and preservative-free artificial tears four times
Table I. Demographic data for the cohort of 80 eyes.

\begin{tabular}{lcc}
\hline Parameter & Mean \pm SD & Range \\
\hline Age (y) & $28.9 \pm 5.5$ & $18-37$ \\
Sex & & \\
Male & $30.5 \pm 6.6$ & $18-37$ \\
Female & $28.2 \pm 5.0$ & $19-37$ \\
Number (n) & & \\
Male & 11 & \\
Female & 29 & \\
\hline
\end{tabular}

$\mathrm{SD}$, standard deviation.

Table II. Summary of the data of the cohort at the pre-operative stage and at 1 year post-operatively $(n=80)$.

\begin{tabular}{llll}
\hline & & 1 Year & \\
Parameter & Pre-operation & post-operatively & P-value \\
\hline Sphere (D) & & & $<0.001$ \\
$\quad$ Mean \pm SD & $-3.96 \pm 1.22$ & $0.21 \pm 0.48$ & \\
Range & $-6.75,-0.50$ & $-1.25,1.25$ & \\
Cylinder (D) & & & $<0.001$ \\
Mean \pm SD & $-0.59 \pm 0.54$ & $-0.29 \pm 0.25$ & \\
Range & $-2.00,0.00$ & $-1.00,0.50$ & \\
SER (D) & & & $<0.001$ \\
Mean \pm SD & $-4.19 \pm 1.35$ & $0.05 \pm 0.46$ & \\
Range & $-7.15,-0.50$ & $-1.38,1.00$ & \\
UCVA (logMAR) & & & $<0.001$ \\
Mean \pm SD & $0.97 \pm 0.26$ & $-0.10 \pm 0.08$ & \\
Range & $0.40,1.50$ & $-0.20,0.10$ & \\
CDVA (logMAR) & & & \\
Mean \pm SD & $-0.10 \pm 0.08$ & $-0.14 \pm 0.07$ & $<0.001$ \\
Range & $-0.20,0.00$ & $-0.20,0.00$ & \\
\end{tabular}

SER, spherical equivalent refraction; UCVA, uncorrected visual acuity; CDVA, corrected distance visual acuity; SD, standard deviation; $\mathrm{D}$, diopters.

daily for six months. The contact lens was removed two to three days after the surgery when corneal re-epithelialization was completed.

Statistical analysis. Statistical analysis was performed using SPSS 19.0 (IBM Corp.). To assess changes in the parameters following surgery, Student's t-test was used to compare pre-operative and post-operative mean values. The change in HOA was calculated as the difference between the post- and pre-operative value. The correlation between the changes in HOA and pre-operative MSER, mean astigmatism and CCAD were tested by calculating the Pearson correlation coefficient. $\mathrm{P}<0.05$ was considered to indicate a statistically significant difference. 


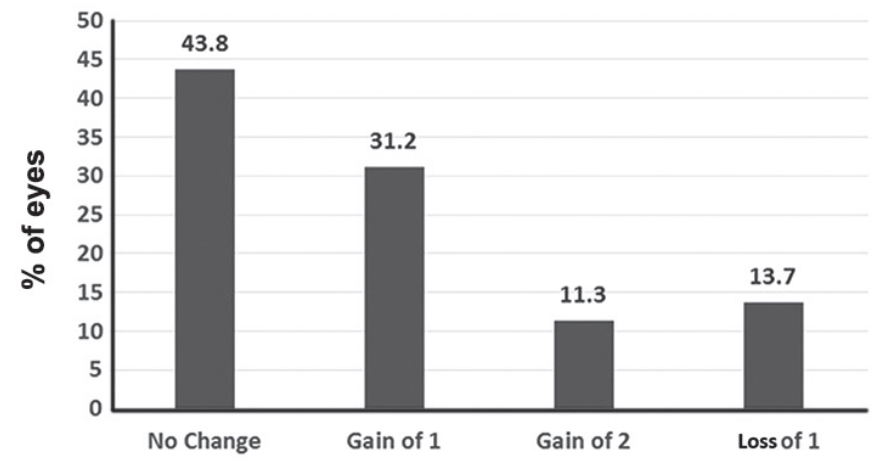

Figure 1. Change in Snellen lines of corrected distance visual acuity.

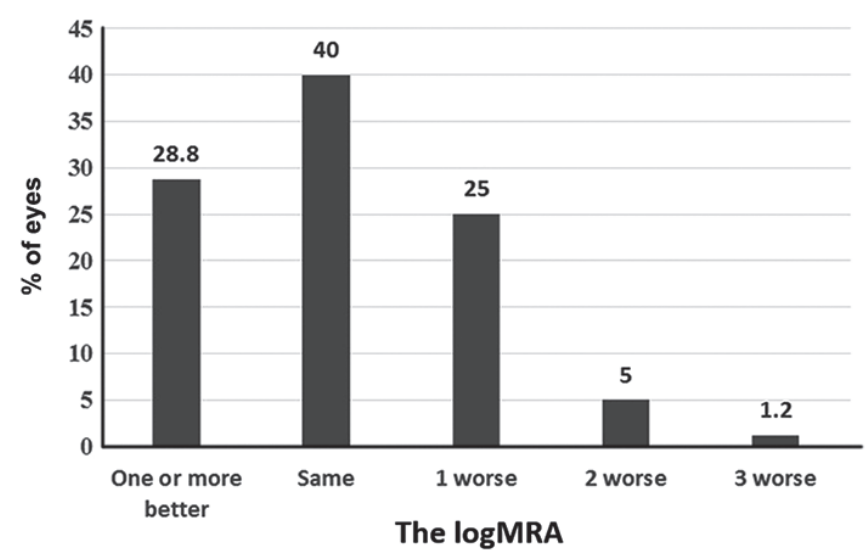

Figure 2. Difference between post-operative uncorrected distance visual acuity and pre-operative corrected distance visual acuity.

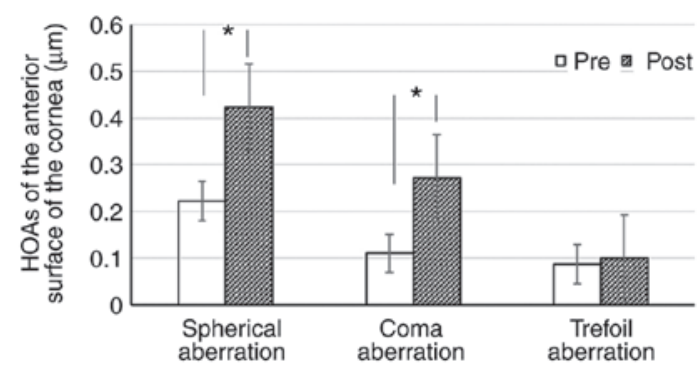

Figure 3. HOAs of the anterior surface of the cornea compared between the pre- and post-operative stages. " $\mathrm{P}<0.01$ vs. pre. HOA, higher-order wavefront aberration; Pre, pre-operatively; Post, post-operatively.

\section{Results}

Patient characteristics. The demographic data of the patients of the present study are listed in Table I. Table II provides a comparative analysis of refractive components (sphere, cylinder, SE refraction) and LogMAR visual acuities prior to and at 1 year after surgery.

$C D V A$ and UDVA. Fig. 1 presents the percentage change in CDVA. The CDVA was increased post-operatively $(\mathrm{P}<0.001)$ in a certain proportion of eyes and remained the same in $43.8 \%$ of the eyes. A total of $31.7 \%$ of eyes gained one Snellen line of CDVA and no eye lost $\geq 2$ lines of CDVA.

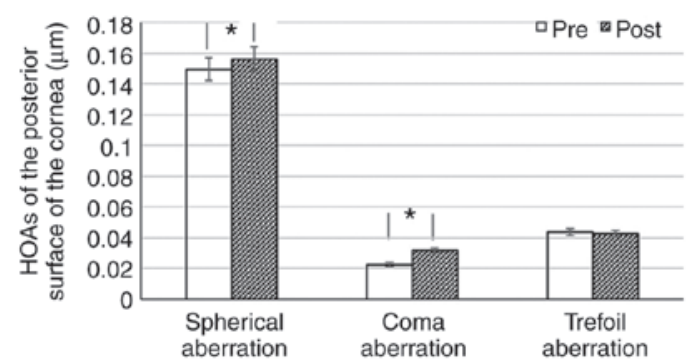

Figure 4. HOAs of the posterior surface of the cornea compared between the pre- and post-operative stages. " $\mathrm{P}<0.01$ vs. pre. HOA, higher-order wavefront aberration; Pre, pre-operatively; Post, post-operatively.

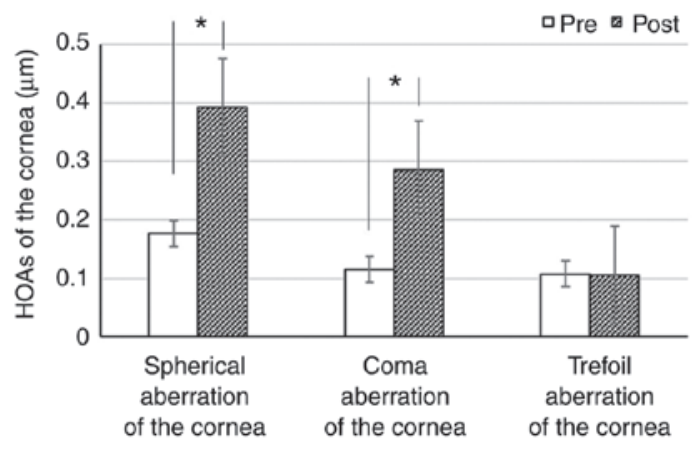

Figure 5. HOAs of the total cornea spherical, coma and trefoil aberrations compared between the pre- and post-operative stages. " $\mathrm{P}<0.01$ vs. pre. HOA, higher-order wavefront aberration; Pre, pre-operatively; Post, post-operatively.

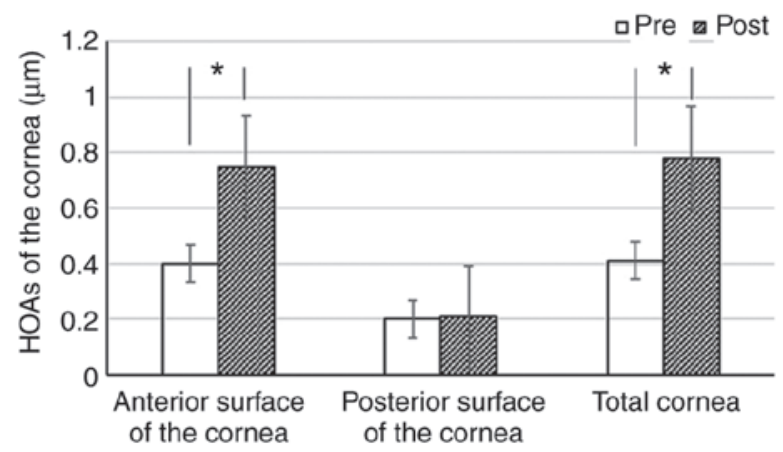

Figure 6. HOAs of the anterior and posterior surface of the cornea and total cornea compared between the pre- and post-operative stages. ${ }^{*} \mathrm{P}<0.01$ vs. pre. HOA, higher-order wavefront aberration; Pre, pre-operatively; Post, post-operatively.

Fig. 2 provides the difference between post-operative UDVA and pre-operative CDVA. The post-operative UDVA was the same or superior compared with the pre-operative CDVA in $\sim 69 \%$ of the eyes.

Changes in HOAs. Table III and Figs. 3-6 present the changes in HOAs of the cornea. Compared with the pre-operative values, the spherical and coma aberration of the anterior (Fig. 3), posterior (Fig. 4) and total cornea (Fig. 5) were significantly increased post-operatively $(\mathrm{P}<0.001)$. By contrast, the trefoil was not significantly affected $(\mathrm{P}=0.262,0.805$ and 0.936 , respectively). The total HOAs of the anterior surface and total cornea were 
Table III. Changes in HOAs of the cornea.

A, Anterior

\begin{tabular}{lcccc}
\hline Measured outcome & Spherical aberration & Coma & Trefoil & HOA root-mean-square \\
\hline Pre-operative & $0.22 \pm 0.08$ & $0.11 \pm 0.08$ & $0.09 \pm 0.07$ & $0.40 \pm 0.09$ \\
Post-operative & $0.42 \pm 0.19$ & $0.27 \pm 0.21$ & $0.10 \pm 0.07$ & $0.75 \pm 0.25$ \\
P-value & $<0.001$ & $<0.001$ & 0.262 & $<0.001$ \\
\hline
\end{tabular}

B, Posterior

\begin{tabular}{lcccc}
\hline Measured outcome & Spherical aberration & Coma & Trefoil & HOA root-mean-square \\
\hline Pre-operative & $0.15 \pm 0.03$ & $0.02 \pm 0.02$ & $0.04 \pm 0.04$ & $0.20 \pm 0.04$ \\
Post-operative & $0.16 \pm 0.03$ & $0.03 \pm 0.03$ & $0.04 \pm 0.03$ & $0.21 \pm 0.06$ \\
P-value & $<0.001$ & $<0.001$ & 0.805 & 0.303 \\
\hline
\end{tabular}

C, Total

\begin{tabular}{lcccc}
\hline Measured outcome & Spherical aberration & Coma & Trefoil & HOA root-mean-square \\
\hline Pre-operative & $0.18 \pm 0.08$ & $0.11 \pm 0.10$ & $0.11 \pm 0.08$ & $0.41 \pm 0.012$ \\
Post-operative & $0.39 \pm 0.20$ & $0.29 \pm 0.22$ & $0.11 \pm 0.08$ & $0.78 \pm 0.26$ \\
P-value & $<0.001$ & $<0.001$ & 0.936 & $<0.001$ \\
\hline
\end{tabular}

HOAs, higher-order wavefront aberrations.

Table IV. Pearson correlation analysis between changes in HOA of the anterior cornea and pre-operative MSER, astigmatism and CCAD.

\begin{tabular}{|c|c|c|c|c|c|c|c|c|}
\hline \multirow[b]{2}{*}{ Parameter } & \multicolumn{2}{|c|}{$\begin{array}{c}\text { Spherical } \\
\text { aberration (post-pre) }\end{array}$} & \multicolumn{2}{|c|}{$\begin{array}{l}\text { Coma } \\
\text { aberration (post-pre) }\end{array}$} & \multicolumn{2}{|c|}{$\begin{array}{c}\text { Trefoil } \\
\text { aberration (post-pre) }\end{array}$} & \multicolumn{2}{|c|}{$\begin{array}{c}\text { HOA } \\
\text { (post-pre) }\end{array}$} \\
\hline & $\mathrm{R}$ & P-value & $\mathrm{R}$ & P-value & $\mathrm{R}$ & P-value & $\mathrm{R}$ & $\mathrm{P}$-value \\
\hline MSER & 0.462 & $<0.001$ & 0.268 & 0.016 & 0.087 & 0.442 & 0.554 & $<0.001$ \\
\hline Astigmatism & 0.191 & 0.090 & 0.260 & 0.020 & -0.279 & 0.012 & 0.312 & 0.005 \\
\hline CCAD & 0.510 & $<0.001$ & 0.323 & 0.004 & 0.014 & 0.901 & 0.583 & $<0.001$ \\
\hline
\end{tabular}

MSER, mean spherical equivalent refraction; CCAD, central corneal ablation depth; HOA, higher-order wavefront aberration.

significantly increased $(\mathrm{P}<0.001)$; however, the posterior surface of the cornea was not significantly changed ( $\mathrm{P}=0.303$; Fig. 6).

Correlations. The correlations between changes in the HOA of the anterior cornea and pre-operative MSER, mean astigmatism and CCAD are presented in Table IV. Significant correlations of the pre-operative MSER, astigmatism and CCAD with the change of coma aberration $(r=0.268, P=0.016 ; r=0.260$, $\mathrm{P}=0.02 ; \mathrm{r}=0.323, \mathrm{P}=0.004$, respectively) and HOA ( $\mathrm{r}=0.554$, $\mathrm{P}<0.001 ; \mathrm{r}=0.312, \mathrm{P}=0.005 ; \mathrm{r}=0.583, \mathrm{P}<0.001$, respectively) of the anterior cornea were determined. The change in spherical aberration of the anterior cornea was significantly correlated with pre-operative MSER $(\mathrm{r}=0.462, \mathrm{P}<0.001)$ and CCAD $(r=0.510, P<0.001)$, but not with astigmatism $(\mathrm{P}=0.09)$.
However, the change in trefoil was only significantly correlated with pre-operative astigmatism $(\mathrm{r}=-0.279, \mathrm{P}=0.012)$.

\section{Discussion}

The present study revealed a significant improvement in SE, astigmatism, UDVA and CDVA in eyes with myopia after one-step TransPRK. Of note, $\sim 96 \%$ eyes had a UDVA of 20/20 or better, with $>95 \%$ treated eyes within $\pm 1.00 \mathrm{D}$ of the intended target refraction and no eye lost two or more Snellen lines of CDVA. It was demonstrated that one-step TransPRK was able to correct myopia effectively. Previous clinical studies also confirmed the efficacy and safety of one-step TransPRK in the correction of myopia $(8,9,13,14)$. 
One other study proved that HOA values of the anterior surface were significantly higher compared with the HOA values of the total cornea (5). According to the pre-operative analysis of wavefront aberration in the present study, the spherical aberration of the anterior corneal surface proved to be significantly higher compared with the spherical aberration of the total cornea. This suggested that the posterior corneal surface may have a compensatory role in the balance of spherical aberration in myopic eyes. Pentacam is a commonly used multi-function instrument for analyzing corneal topography in patients at the pre-operative stage. It may also be used to analyze HOAs of patients' corneas. The use of Pentacam to analyze the HOAs of the patients prior to and after surgery is of clinical guiding significance.

In the present study, the spherical and coma aberration of the anterior, posterior and total cornea were significantly increased post-operatively. Compared with the pre-operative values, the trefoil aberration was not significantly affected, nor were the HOAs of the posterior corneal surface. Induced HOAs after one-step TransPRK may be attributed to various factors. A certain extent of decentration during keratorefractive surgery, fluctuations in accommodation and changes of tear film after surgery have been reported in the induction of HOAs (7,15). By using very high-frequency digital ultrasonography, Reinstein et al (4) determined that the corneal epithelium was thicker inferiorly than superiorly and thicker nasally than temporally. Unequal pre-operative epithelial thickness may potentially be a source of HOAs. Another factor for the increase of HOAs may be the changes in epithelial thickness after surgery. Hou et al (16) assessed the characteristics of epithelial thickening, indicating that the epithelium was thicker inferiorly than superiorly and temporally than nasally after TransPRK. Following PRK, Chen et al (17) observed a maximum amount of epithelial thickening temporally.

The present study analyzed the correlation between changes in corneal HOAs and pre-operative diopter and depth of cut, which is significant to predict the changes in post-operative corneal HOAs. The increase in HOAs of the anterior cornea was linearly correlated with the pre-operative MSER, the mean astigmatism and the CCAD. Pre-operative astigmatism was significantly associated with the post-operative increases in trefoil aberration but not with spherical aberration. According to these results, the diopter of myopia and the ablation depth should be considered as important factors for increased HOAs. In patients with a higher MSER prior to surgery, the chances of increased HOAs after surgery are increased. This may be one of the factors affecting the visual quality following myopia surgery. The biomechanical response of the cornea tissue may be changed after the surgery, which may then induce HOAs to a certain extent. Astigmatism may have an increased effect on the trefoil. In patients with high astigmatism refraction, the trefoil should be monitored post-operatively.

In conclusion, the present study demonstrated the effectiveness of TransPRK for the treatment of myopia. The total HOAs of the anterior surface of the cornea and further aberrations, including spherical and coma aberrations, were increased post-operatively. The increased HOAs were linearly correlated with the degree of pre-operative myopia and CCAD. The increase in the trefoil was only significantly correlated with the degree of pre-operative astigmatism. There is a requirement for further study of the predictability of the treatment algorithm used in one-step TransPRK.

\section{Acknowledgements}

Not applicable.

\section{Funding}

No funding was received.

\section{Availability of data and materials}

The datasets used and/or analyzed during the present study are available from the corresponding author on reasonable request.

\section{Authors' contributions}

LX performed the study, participated in the design, drafted the manuscript, revised the manuscript and gave final approval of the version for publication.

\section{Ethics approval and consent to participate}

The study was performed in accordance with the Declaration of Helsinki and was approved by the Ethics Committee of the Peking University International Hospital (Beijing, China). Patients included in the present study had complete clinical data and cooperated with the medical staff to complete the relevant medical treatment. Written informed consent was obtained from the patients and/or their guardians.

\section{Patient consent for publication}

Not applicable.

\section{Competing interests}

The author declared that he has no competing interests.

\section{References}

1. Clinch TE, Moshirfar M, Weis JR, Ahn CS, Hutchinson CB and Jeffrey JH: Comparison of mechanical and transepithelial debridement during photorefractive keratectomy. Ophthalmology 106: 483-489, 1999.

2. Arba Mosquera S and Awwad ST: Theoretical analyses of the refractive implications of transepithelial PRK ablations. Br J Ophthalmol 97: 905-911, 2013.

3. Ghadhfan F, Al-Rajhi A and Wagoner MD: Laser in situ keratomileusis versus surface ablation: Visual outcomes and complications. J Cataract Refract Surg 33: 2041-2048, 2007.

4. Reinstein DZ, Archer TJ, Gobbe M, Silverman RH and Coleman DJ: Epithelial thickness in the normal cornea: Three-dimensional display with Artemis very high-frequency digital ultrasound. J Refract Surg 24: 571-581, 2008.

5. Juhasz E, Kranitz K, Sandor GL, Gyenes A, Toth G and Nagy ZZ: Wavefront properties of the anterior and posterior corneal surface after photorefractive keratectomy. Cornea 3: 172-176, 2014.

6. Serrao S, Lombardo G, Ducoli P and Lombardo M: Long-term corneal wavefront aberration variations after photorefractive keratectomy for myopia and myopic astigmatism. J Cataract Refract Surg 37: 1655-1666, 2011.

7. Lee SB, Hwang BS and Lee J: Effects of decentration of photorefractive keratectomy on the induction of higher orderwavefront aberrations. J Refract Surg 26: 731-743, 2010. 
8. Adib-Moghaddam S, Soleyman-Jahi S, Salmanian B, Omidvari AH, Adili-Aghdam F, Noorizadeh F and Eslani M: Single-step transepithelial photorefractive keratectomy in myopia and astigmatism: 18-month follow-up. J Cataract Refract Surg 42: 1570-1578, 2016 .

9. Kaluzny BJ, Cieslinska I, Mosquera SA and Verma S: Single-step transepithelial PRK vs Alcohol-assisted PRK in myopia and compound myopic astigmatism correction. Medicine (Baltimore) 95: e1993, 2016.

10. Aslanides IM, Padroni S, Arba Mosquera S, Ioannides A and Mukherjee A: Comparison of single-step reverse transepithelial all-surface laser ablation (ASLA) to alcohol-assisted photorefractive keratectomy. Clin Ophthalmol 6: 973-980, 2012.

11. Miháltz K, Kovács I, Takács A and Nagy ZZ: Evaluation of keratometric, pachymetric, and elevation parameters of keratoconic cornea with pentacam. Cornea 28: 976-980, 2009.

12. Bastawrous A, Silvester A and Batterbury M: Laser refractive eye surgery. BMJ 342: d2345, 2011.

13. Aslanides IM, Georgoudis PN, Selimis VD and Mukherjee AN1: Single-step transepithelial ASLA (SCHWIND) with mitomycin-C for the correction of high myopia: Long term follow-up. Clin Ophthalmol 9: 33-41, 2014.
14. Luger MH, Ewering T and Arba-Mosquera S: Myopia correction with transepithelial photorefractive keratectomy versus femtosecond assisted laser in situ keratomileusis: One-year case-matched analysis. J Cataract Refract Surg 42: 1579-1587, 2016.

15. Artal P, Chen L, Fernández EJ, Singer B, Manzanera S and Williams DR: Adaptive optics for vision: The eye's adaptation to point spread function. J Refract Surg 19 (Suppl): S585-S587, 2003.

16. Hou J, Wang Y, Lei Y, Zheng X and Zhang Y: Corneal epithelial remodeling and its effect on corneal asphericity after transepithelial photorefractive keratectomy for myopia. J Ophthalmol 2016: 8582362, 2016.

17. Chen X, Stojanovic A, Liu Y, Chen Y, Zhou Y and Utheim TP: Postoperative changes in corneal epithelial and stromal thickness profiles after photorefractive keratectomy in treatment of myopia. J Refract Surg 31: 446-453, 2015.

(i) (9) This work is licensed under a Creative Commons Attribution-NonCommercial-NoDerivatives 4.0 International (CC BY-NC-ND 4.0) License. 